\title{
Variedad de la presentación citogenética en el síndrome de Turner, prenatal y postnatal
}

\author{
Wilmar Saldarriaga ${ }^{1,4,6}$ Mariana Valencia ${ }^{1}$, Andrés Fandiño-Losada ${ }^{1,3}$, Fabián Andrés \\ Ruiz $^{1,2}$, Carolina Isaza. $1,4,5$ \\ ${ }^{1}$ Universidad del Valle, Cali, Colombia, Grupo de Investigación en Malformaciones Congénitas Perinatales y Dismorfología \\ de la Universidad del Valle, MACOS; ${ }^{2}$ Grupo Estudiantil Clinical Genetics Group, CGG-Universidad del Valle, Cali, Colom- \\ bia; ${ }^{3}$ Escuela de Salud Pública, Facultad de Salud, Universidad del Valle, Cali, Colombia; ${ }^{4}$ Departamento de Morfología, \\ Escuela de Ciencias Básicas, Facultad de Salud, Universidad del Valle, Cali Colombia; ${ }^{5}$ Laboratorio de Genética Médica \\ "Carolina Isaza", Clínica DIME, Cali, Colombia; ${ }^{6}$ Departamento de Ginecología y Obstetricia, Escuela de Medicina, Univer- \\ sidad del Valle, Cali, Colombia.
}

\section{RESUMEN}

Antecedentes: El síndrome de Turner (ST) es causado por la ausencia total o parcial del cromosoma X y posee una gran variedad en su presentación citogenética. Objetivos: Determinar la variedad de presentación citogenética y la existencia de diferencias entre los casos diagnosticados in útero y los de diagnóstico postnatal, en pacientes con ST en dos laboratorios de referencia de Cali, Colombia. Métodos: Se realizó un estudio observacional descriptivo de corte transversal, se incluyeron pacientes con diagnóstico de ST, cuyo cariotipo se realizó entre los años 2000 y 2012, en los laboratorios de citogenética de la Universidad del Valle y un instituto de genética de Cali, Colombia. Se recolectó información del reporte del cariotipo, tipo de muestra y tiempo de realización del diagnóstico y se determinó frecuencias y asociaciones estadísticas entre las variables a estudiar. Resultados: Se incluyeron 181 pacientes con fórmula cromosómica compatible con ST; 69 fueron diagnosticados in útero, los demás, en recién nacidos vivos, infantes o adultos. La fórmula cromosómica 45 X0 se encontró en el $95,6 \%$ de los casos de diagnóstico prenatal y $58 \%$ de los de diagnóstico postnatal. Se aplicó la prueba del test exacto de Fisher, comparando los múltiples subgrupos de la variedad de presentación citogenética de diagnóstico prenatal y postnatal, encontrándose diferencias estadísticamente significativas en la distribución de las dos poblaciones evaluadas $(p<0,001)$. Conclusiones: Existen diferencias significativas en los cariotipos de los pacientes con ST diagnosticados in útero, respecto a los diagnosticados en vida extrauterina. Se postula que esa diferencia tendría una explicación biológica sobre la posibilidad de muerte in útero por la ausencia total del cromosoma X.

\section{PALABRAS CLAVE: Síndrome de Turner, citogenética, cariotipo anormal, alteración cromosómica, consejería genética}

\section{SUMMARY}

Background: Turner syndrome is caused by the complete or partial absence of chromosome $\mathrm{X}$ and has a great variety in their cytogenetic presentation. Objectives: To determine the variety of cytogenetic presentation and the presence of differences between cases diagnosed in uterus and postnatally, in patients with Turner syndrome at two reference laboratories of Cali, Colombia. Methods: A descriptive cross-sectional study was performed. We included patients with cytogenetic diagnosis of Turner syndrome performed between 2000 and 2012 at cytogenetic laboratories of Universidad del Valle and a genetic institute in Cali, 
Colombia. The information of karyotype result, type of sample and the diagnosis moment was collected, determining frequencies and statistical associations. Results: 181 patients with Turner's chromosomic presentation; 69 were diagnosed in uterus, the other as live newborns, infants or adults. Chromosomal formula $45 \mathrm{X} 0$ was found in $95.6 \%$ of cases with prenatal diagnosis and $58 \%$ of postnatal diagnosis. Fisher's test was applied, comparing the cytogenetic presentations of prenatal and postnatal diagnosis, statistically significant difference in the distribution of the two populations evaluated was found $(p<0.001)$. Conclusions: There are significant differences in the karyotypes of patients with ST diagnosed in utero, compared to those diagnosed in postnatal life. We hypothesize that this difference would have a biological explanation due to a higher probability of death in utero by the complete absence of chromosome $\mathrm{X}$.

\section{KEY WORDS: Turner syndrome, cytogenetics, abnormal karyotype, chromosome alterations, genetic counseling}

\section{INTRODUCCIÓN}

El Síndrome de Turner (ST) es una enfermedad genética causada por la ausencia completa o parcial del cromosoma $X$ y en ocasiones por mosaicismos; los pacientes con este síndrome se caracterizan por la triada: talla baja, amenorrea primaria y otras anomalías como cuello alado y/o corto y cúbitus valgus (1).Para el diagnóstico prenatal, se debe sospechar la patología, en los casos en que en la ecografía obstétrica se observa higroma quístico, hidrops no inmune o la presencia de otras anormalidades anatómicas inespecíficas como cardiopatías y restricción de crecimiento intrauterino $(2,3)$.

Afecta 1 de 2.000 a 5.000 recién nacidos de sexo femenino y se estima que el $3 \%$ de las concepciones de embriones y fetos femeninos tienen esta patología, llegándose a abortar espontáneamente en el primer trimestre entre el 95 al $99 \%(4,5,6)$.

EI ST tiene una variación importante en su presentación citogenética. En el $50 \%$ de los casos hay ausencia total del cromosoma $\mathrm{X}$, presentando un complemento cromosómico $45, \mathrm{X0}$; el otro $50 \%$ de los casos tienen múltiples presentaciones cromosómicas como mosaicismos, deleciones parciales o translocaciones, cromosomas en anillo o re-arreglos complejos $(5,6,7)$.

La importancia de identificar las diferentes presentaciones citogenéticas radica en que la presencia de anomalías estructurales del cromosoma $X$ mosaicos para una línea celular $46, X X$, son consideradas como un factor protector para el feto, al reducir la mortalidad fetal y la severidad del fenotipo, al compararse con la monosomía completa del cromosoma $X(6,7,8)$; a la vez que la presencia del cromosoma $Y$ predispone a la aparición de un raro tumor, el gonadoblastoma, hasta en un $30 \%$ de los pacientes (8-11).

En Colombia hace más de 20 años se determi- nó las frecuencias de las diferentes presentaciones citogenéticas de síndrome de Turner; Giraldo y cols (12) revelaron que en los 38 casos estudiados, la mitad tenían la típica monosomía del cromosoma $X$ y los demás, mosaicismo y en menor medida, alteraciones estructurales y morfológicas del cromosoma $\mathrm{X}$; resultados acordes con las frecuencias determinadas en la literatura mundial. Sin embargo desde la fecha no se tienen nuevos estudios de población Colombiana con un número de muestra significativo, que evidencie la proporción actual de las presentaciones citogenéticas del ST.

Los objetivos de este estudio son encontrar la variedad de presentación citogenética en pacientes diagnosticados con ST en dos laboratorios de referencia de la ciudad de Cali y determinar si existen diferencias en la presentación citogenética entre los casos diagnosticados in útero y los de diagnóstico postnatal.

\section{METODOLOGÍA}

Se realizó un estudio observacional descriptivo de corte transversal. Se incluyeron los resultados decariotipos con diagnóstico de ST obtenidos de los laboratorios de citogenética del Instituto de Genética Medica "Carolina Isaza" de la Clínica DIME y de la Universidad del Valle, Cali, Colombia, entre el 1 de enero de 2000 y el 31 de diciembre de 2012. Se definió diagnóstico de ST, como todo resultado en paciente femenino que tuviera cualquiera de las alteraciones cromosómicas del $\mathrm{X}$, incluyendo ausencia total, pérdidas de fragmentos del cromosoma, mosaicismos, isocromosomas, cromosomas en anillo y otras presentaciones menos comunes.

Se recolectó información retrospectiva del reporte de resultados del cariotipo de cada paciente. Se registró la presentación citogenética (resultado de cariotipo). 
Tipo de muestra: sangre periférica (niños o adultos), líquido amniótico, vellosidad corial o sangre de cordón umbilical (in útero); se clasificó el momento de toma de la muestra como prenatal o postnatal. Se excluyeron los casos con datos incompletos, informe confuso o ilegible y pacientes de sexo masculino.

Los datos fueron consignados en un formato de recolección elaborado por los investigadores, las distintas presentaciones citogenéticas se consignaron en grupos con características similares, siguiendo los lineamientos de la literatura internacional. Las variables cuantitativas fueron analizadas mediante tablas y gráficas tabuladas en Microsoft ExcelC y posteriormente analizada en el paquete estadístico Stata $11 \odot$, determinándose frecuencias y asociaciones estadísticas entre las variables a estudiar.

Se revisó la literatura internacional, utilizando las bases de datos de PubMed, HINARI, OVID, ScienceDirect y Scielo, se seleccionaron aquellos artículos que tuviesen como mínimo 50 casos en diagnóstico prenatal ó 100 casos en diagnóstico posnatal, en los estudios que no diferenciaran el momento del diagnóstico se estableció un número mínimo de 150 casos, además de un único trabajo previamente publicado en Colombia. A las series de casos de estos trabajos se aplicó la prueba de $x^{2}$ (chi cuadrado), en dos ocasiones: comparando con la totalidad de los casos de nuestro reporte e incluyendo solamente los casos diagnosticados pre o posnatalmente según el caso. El estudio contó con el aval del comité de ética humana de la Universidad del Valle y de la Clínica DIME.

\section{RESULTADOS}

Durante el periodo estudiado se encontraron 181 casos que cumplían los criterios de inclusión, con cariotipos positivos para un diagnóstico compatible con alguna presentación citogenética de ST. En el $38 \%$ de los casos $(n=69)$, la muestra fue tomada prenatalmente: de líquido amniótico (LA) en 58 casos, biopsia de vellosidad corial (BVC) en 7 casos y un único caso con toma de sangre fetal de cordón umbilical. Para las muestras de los 112 casos restantes, se tomó sangre periférica, posnatal. La variedad de presentación citogenética del total de los casos incluidos, diferenciados en los subgrupos de diagnóstico prenatal y postnatal, se presenta en la Tabla I. Se aplicó la prueba del test exacto de Fisher, comparando los subgrupos, de diagnóstico prenatal y de diagnóstico postnatal, encontrándose diferencias estadísticamente significativas en la distribución de las dos poblaciones evaluadas (Fisher: $p<0,001$ ).

\section{Series de diagnóstico de síndrome de Turner.}

Se incluyeron 3 artículos que cumplieron los criterios de inclusión; los trabajos de Syberty cols (7) y Saengel y cols (1) que cuentan con pacientes con diagnóstico posnatal y el de Papp y cols (3) solo con diagnóstico prenatal. También se incluyó un trabajo realizado con población colombiana con diagnóstico posnatal en los años 80's de Giraldo y cols (12) (Tabla I). Se les aplicó las pruebas de Chi cuadrado a estas series, en dos ocasiones a cada una, comparando con: 1) la totalidad de los casos de nuestro reporte y, 2) incluyendo solamente los casos diagnosticados pre o posnatalmente según el caso.

Se aplicó la prueba de Chi cuadrado a las series de casos seleccionadas, para determinar si correspondían a series independientes entre sí, no encontrándose diferencias estadísticamente significativas. Hubo diferencias significativas al comparar los casos reportados en este artículo con los presentados por Sybert, Saengel, Papp y Giraldo. Se aplicó el test exacto de Fisher comparando los resultados reportados en los subgrupos de posnatal vs. Sybert, Saengel y Giraldo (series de casos postnatales) y prenatal vs. Papp (que incluye solo casos de diagnóstico prenatal), hallándose diferencias significativas $(p<0,05)$ solamente en la comparación con la serie de Papp.

\section{DISCUSIÓN}

En el síndrome de Turner se observan diferentes presentaciónes citogenéticas, siendo la más frecuente, la presentación "clásica" de monosomía del cromosoma X o comúnmente conocida como "X0"; sin embargo, en la serie mas grande encontrada en la literatura revisada, se observa que esta presentación solo se encuentra en el $45 \%$ de los casos (7). De esta manera el ST, marca diferencia con otros síndromes causados por alteraciones cromosómicas como el síndrome de Down, donde la fórmula clásica de trisomía 21 se encuentra en el $95 \%$ de los pacientes $(13,14,15)$; o los síndromes de Patau y Edwards en los cuales su fórmula cromosómica más frecuente (trisomía 13 o 18) se presenta en el $80 \%$ de los casos $(16,17)$. Esto tiene consecuencias en el pronóstico de los casos y en la consejería genética y reproductiva de los padres $(18,19)$. 


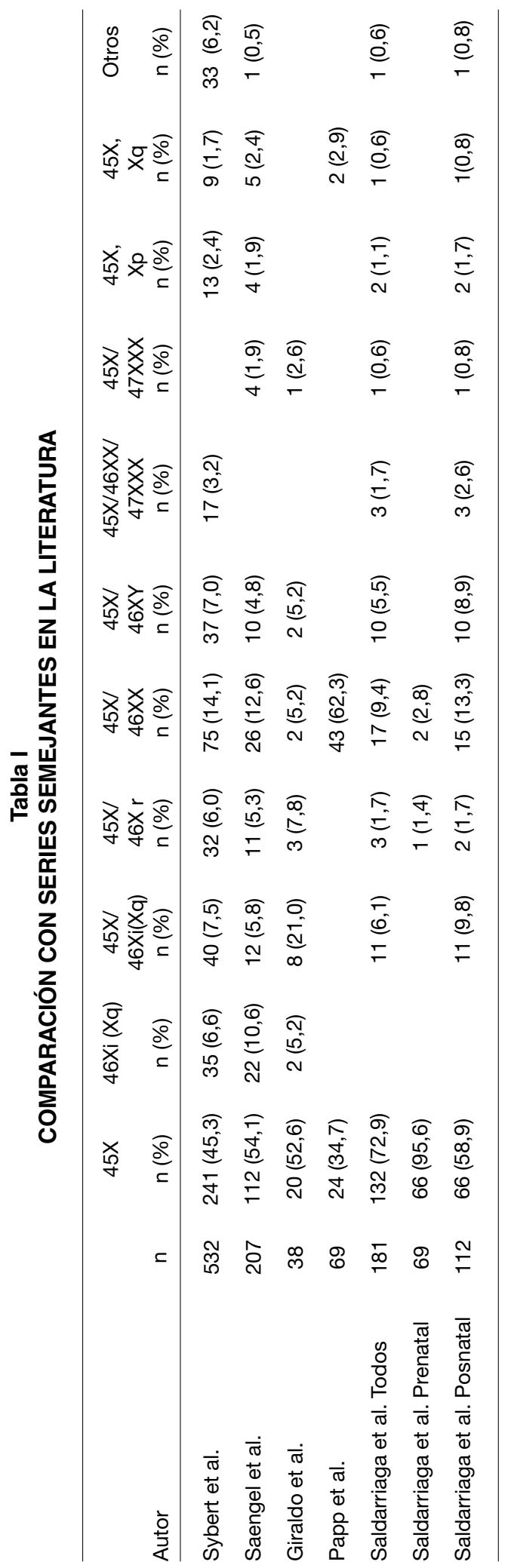


En el presente estudio se encontró, que del total de los pacientes incluidos, el $73 \%$ tuvieron la presentación citogenética tradicional (X0); al comparar estos datos con los resultados de otras series de la literatura, se encontraron diferencias estadísticamente significativas en las frecuencias relativas de las presentaciones cromosómicas. Esta diferencia puede explicarse por que este estudio contiene casos de diagnóstico pre y posnatal; a su vez, el importante aporte de pacientes con diagnóstico prenatal con X0 (95\%), al total de casos produciría la diferencia con estudios donde el diagnóstico fue exclusivamente posnatal.

Al clasificar los pacientes en subgrupos, dependiendo del momento de realización del diagnóstico (pre y postnatal) encontramos que el $58 \%$ en los casos con diagnóstico posnatal, presentaban la formula cromosómica $45 \mathrm{X0}$, al comparar este hallazgo con la literatura revisada, donde solo se incluyeran casos de diagnóstico posnatal; se observó que la frecuencia de dicha presentación es similar, así: Sybert $45 \%$, Saenger $54 \%$, Giraldo $52 \%$, diferencias no significativas.

Al comparar los resultados aquí encontrados en el subgrupo de casos con diagnóstico prenatal, con los reportados por Papp en el 2006 (3), quien reporta únicamente casos de diagnóstico prenatal, encontramos que la frecuencia de la formula cromosómica $45 \times 0$, es de $95 \%$ vs $34 \%$, respectivamente. Siendo una diferencia amplia y estadísticamente significativa que podría ser explicada por diferencias en la población estudiada; probablemente en nuestra población la indicación de los procedimientos invasivos para realizar el cariotipo permitió el diagnóstico de anomalías fetales de mayor complejidad. Sin embargo ninguno de los dos estudios aportó los datos para aclarar este factor.

Al comparar nuestros resultados, según diagnóstico pre y posnatal, se encontró que la fórmula citogenética X0 correspondió al $95 \%$ y $58 \%$ de los casos, respectivamente, diferencias estadísticamente significativas. Esto se debe, a que pacientes con ST, que presentan la fórmula XO, tiene mayor expresión fenotípica, presentando malformaciones complejas y mayor mortalidad in útero, entre el 95 y el $99 \%$ de los casos de diagnóstico prenatal mueren antes del nacimiento, evidenciando la gravedad de esta presentación citogenética $(7,11,20,21,22)$. Se considera que los hallazgos fenotípicos se pueden evidenciar con mayor facilidad en la ecografía obstétrica, tras la cual se genera la sospecha de cromosomopatía y se justifica la realización de una prueba invasiva como biopsia de vellosidad co- rial, amniocentesis o cordocentesis $(20,21,22)$. Al contrario de los casos con ST diagnosticado posnatalmente, donde una proporción considerable tendrían una fórmula cromosómica diferente de X0, sugiriendo que las otras presentaciones tiene manifestaciones menos graves para la sobrevida de estos pacientes.

El estudio, manejo y tratamiento del ST, representa un reto para los médicos y en particular para los especialistas en ginecología y obstetricia, que deberán enfrentarse a la patología en diferentes escenarios, donde resaltan parejas con resultado de un cariotipo obtenido in útero, en el contexto del control prenatal o pacientes que consultan por amenorrea primaria o alteraciones Müllerianas; deberá entonces interpretar el resultado del cariotipo, considerando las complicaciones y la severidad del cuadro clínico al presentar la clásica monosomía del cromosoma $\mathrm{X} \mathrm{o}$ alguna de las variantes ya descritas. Se debe prestar especial atención a los pacientes que presenten en su cariotipo un cromosoma $Y$ en alguna línea celular, pues este hallazgo tiene gran relación con la aparición de un extraño tumor, el gonadoblastoma, en el $30 \%$ de los casos $(7,8,23,24)$.

Al considerar el impacto que la presentacióncitogenética del ST tiene en el cuadro clínico, se puede dar un mejor manejo y pronóstico a lapaciente, a la vez que se brinda a los familiares una adecuada consejería genética, con aclaración de posibilidad de repetición y extensión de estudios citogenéticos a los padres $(1,7,19)$.

\section{CONCLUSIONES}

Se aporta un estudio con un número considerable de casos de ST, encontrándose que existen diferencias significativas en los cariotipos de los pacientes diagnosticados in útero, con respecto a los diagnosticados en vida extrauterina. Se postula que esa diferencia tendría una explicación biológica sobre la posibilidad de muerte in útero por la ausencia total del cromosoma X. La presentación citogenética del ST tiene gran importancia en la práctica médica y gineco-obstétrica, pues influye directamente sobre el pronóstico de morbilidad y mortalidad de fetos, niños y adolescentes que asisten a consulta con diagnóstico de cariotipo de síndrome de Turner. Se debe dar un manejo especial a pacientes que presentan un cromosoma $Y$ dada su asociación con gonadoblastoma. Además, para cada variante de la presentación citogenética existe una consejería genética específica que se debe realizar idealmente en conjunto con un genetista. 
Agradecimientos: A los laboratorios de citogenética del departamento de Morfologíade la Universidad del Valle y del Instituto de Genética Médica "Carolina Isaza" por facilitar la información.

\section{REFERENCIAS}

1. Saengel P. Turner syndrome. En: Sperling M., Editor. Pediatrics endocrinology. 3rd Edition. Philadelphia: Elsevier, 2008. p. 610-61.

2. Chuchracki M, Szczepaniak A, Sedziak A, et al. Frequency of prevalence of Turner syndrome in fetuses of patients referred to genetic amniocentesis in 20072011. PrzeglLek 2012;69(10):1011-4.

3. Papp C, Beke A, Mezei G, et al. Prenatal diagnosis of Turner syndrome: report on 69 cases. J Ultrasound Med 2006;25(6):711-7; 718-20.

4. Hsu L. Prenatal diagnosis of chromosomal abnormalities through amniocentesis. En: Milunsky A (Ed). Genetic disorder and the fetus. Baltimore: John Hopkins University Press; 1998. p. 179-248.

5. Turner C, Dennis NR, Skuse NH, Jacobs PA. Seven ring $(X)$ chromosomes lacking XIST locus, six with an unexpectedly mild phenotype. Hum Genet 2000;106:93-100.

6. Ranke M, Saenger P. Turner's syndrome. Lancet 2001;358:309-14.

7. Sybert V, McCauley E. Turner's syndrome. N Engl J Med 2004;351:1227-38.

8. Pinsker JE. Turner syndrome: Updating the paradigm of clinical care. J ClinEndocrinolMetab 2012;97:9941003.

9. Chacko E, Graber E, Molly O, et al. Update on Turner and Noonan syndromes. EndocrinolMetabClin N Am 2012;41:713-34.

10. Saldarriaga W, Sánchez F, Isaza C. Síndrome de Turner con mosaicismo 45X/46XY. Reporte de caso. RevChilObstetGinecol 2011;76:47-51.
11. Stochholm K, Juul S, Juel K, et al. Prevalence, incidence, diagnostic delay, and mortality in Turner syndrome. J ClinEndocrinolMetab 2006;91:3897-902.

12. Giraldo A, Silva E, Bueno ML, et al. Estudio citogenético de 38 pacientes con síndrome de Turner. Biomédica 1983;3:104-17.

13. Megarbane A, Ravel A, Mircher C, et al. The 50th anniversary of the discovery of trisomy 21 : The past, present, and future of research and treatment of Down syndrome. Genet Med 2009;11:611-6.

14. Online Mendelian Inheritance in Man, OMIM®. Johns Hopkins University, Baltimore, MD. MIM Number: \{\#190685 \}: \{10/25/2013\}:http://omim.org/ Acceso el 20 de enero de 2014.

15. Mikkelsen M. Down's syndrome cytogenetic epidemiology. Hereditas 1977;86:45-59.

16. Careda A, Carey J. The trisomy 18 syndrome. Orphanet J Rare Dis 2012;7:81.

17. Hassold T, Jacobs P A, Leppert M, Sheldon M. Cytogenetic and molecular studies of trisomy 13. J Med Genet 1987;24:725-32.

18. Barber JCK,McKinlay GRJ, Sutherland GR, Shaffer LG. Chromosome abnormalities and genetic counselling. Human Genet2012;131:1393-4.

19. Verp MS, Bombard AT, Simpson JL, Elias S, Reynolds JF, Hall JG. Parental decision following prenatal diagnosis of fetal chromosome abnormality. Am J Med Genet 1988;29:613-22.

20. Chuchracki M, Szczepaniak A, Sedziak A, et al. Frequency of prevalence of Turner syndrome in fetuses of patients referred to genetic amniocentesis in 20072011. PrzeglLek2012;69:1011-4.

21. Gravholt $\mathrm{CH}$, Juul S, Naeraa RW, et al. Prenatal and postnatal prevalence of Turner's syndrome: a registry study. BMJ 1996;312:16-21.

22. Grayholt $\mathrm{CH}$, Juul S, Naeraa RW, Hansen J. Morbidity in Turner Syndrome. J ClinEpidemiol 1998;51:147-58.

23. Grayholt CH. Medical problems of adult Turner's syndrome. Horm Res 2001;56 Suppl 1:44-50.

24. Ostberg JE, Conway GS. Adulthood in women with Turner syndrome. Horm Res 2003;59:211-21. 